www.jmscr.igmpublication.org

Impact Factor (SJIF): 6.379

Index Copernicus Value: 79.54

ISSN (e)-2347-176x ISSN (p) 2455-0450

crossrefDOI: https://dx.doi.org/10.18535/jmscr/v6i10.186

Journal Of Medical Science And Clinical Research

IGM Publication

An Official Publication of IGM Publication

\title{
Association of non-Alcoholic Fatty Liver Disease with Coronary Artery Disease Risk Factors and between Severity of non-Alcoholic Fatty Liver Disease with Coronary Artery Disease among type II Diabetes Mellitus Reported at Tertiary care Hospital, Jaipur
}

Authors

\author{
Krishan Kumar Meena ${ }^{1}$, Rajveer Bainiwal*2, Sudhir Bhandari ${ }^{3}$, Vishal Gupta ${ }^{4}$ \\ ${ }^{1,2}$ Registrar, Department of Medicine, S.M.S. Medical College \& Attached Hospitals, Jaipur \\ ${ }^{3}$ Principal and Controller and Senior Professor, Department of Medicine, S.M.S. Medical College \& \\ Attached Hospitals, Jaipur \\ ${ }^{4}$ Assistant Professor Department of Medicine S.M.S. Medical College \& Attached Hospitals, Jaipur \\ * ${ }^{*}$ Corresponding Author \\ Rajveer Bainiwal
}

Registrar, Department of Medicine, S.M.S. Medical College \& Attached Hospitals, Jaipur, India

\begin{abstract}
Background: Non-alcoholic fatty liver disease (NAFLD) has proven strong association with metabolic syndrome and it can be associated with coronary artery disease risk factors. This study was conducted to establish the association between non-alcoholic fatty liver disease and coronary artery disease risk factors and assessing the relationship between severity of NAFLD and presence of coronary artery disease $(C A D)$ in patients of type 2 diabetes mellitus. So that it can be used as tool in preventing future CAD events in type 2 diabetic patients.

Methods: Ultrasonography was used to detect fatty liver among 340 type 2 diabetic subjects. The study group was divided into two groups: Type 2 diabetes mellitus patients with NAFLD and Type 2 diabetes mellitus patients without NAFLD. On selection, proper history and clinical examination were done regarding demographics, cardiovascular, hepatic and renal disease, drug use, smoking and alcohol consumption. Presence of coronary artery disease was defined by history of CAD medications, ECG changes, echocardiographic findings. Qualitative data expressed as number and percentage or proportion and analyzed using chi-square test. Quantitative data will be expressed as mean \pm S.D and analyzed using unpaired t-test and statistical significance will be set at $P<0.05$. Statistical analysis was done using software Epi info version 7.2.1.0.

Results: Coronary artery disease and its risk factors were more prevalent in NAFLD group than in Non NAFLD group. Occurrence of CAD in NAFLD patients is grade dependent i.e. as the severity of NAFLD increases the chances of occurrence of CAD also increases $(p<0.001)$.

Conclusion: Presence of NAFLD in the type 2 diabetes patients can be associated with coronary artery disease risk factors and occurrence of CAD among type 2 diabetes patients with NAFLD depends on severity of fatty liver.
\end{abstract}

Keywords: Non-Alcoholic Fatty Liver Disease; Coronary Artery Disease; Type 2 Diabetes mellitus. 


\section{Introduction}

Non-alcoholic fatty liver disease (NAFLD) is a distinct hepatic condition characterized by abnormal fat accumulation in liver cells, mostly in the form of triglycerides, which exceed $5 \%$ of liver weight; resembling alcoholic liver damage histologically. Non-alcoholic fatty liver disease (NAFLD) appears to be the hepatic manifestation of metabolic syndrome, and is increasingly recognized as a major cause to the occurrence of chronic liver disease world-wide. NAFLD has increased with the epidemics of obesity and type 2 diabetes mellitus ${ }^{1}$. The term NAFLD is used to describe a variety of fatty liver changes ranging from simple fat accumulation to steato-hepatitis, cirrhosis and hepatocellular carcinoma (HCC), in the absence of any history of excessive alcohol intake $^{2}$. In the last decade, several studies have demonstrated independently a strong association between NAFLD and each component of the metabolic syndrome, including central obesity, hypertriglyceridemia and mixed hyperlipidaemia, type 2 diabetes mellitus and hypertension. Increased NAFLD prevalence $(57.5-74 \%)$ in obese patients has been reported ${ }^{3}$. Dyslipidaemia and insulin resistance both are strongly associated with the prevalence of NAFLD. Prevalence of hypertriglyceridemia in different NAFLD case series is quite variable, but all evidence linked it to NAFLD. Recent evidence suggests that NAFLD should be considered as the hepatic manifestation of metabolic syndrome. ${ }^{4}$

\section{Materials and Methods}

It was a Hospital based observational study conducted over a period of one year at a tertiary care hospital in Jaipur. There were 340 subjects in the study as per the inclusion criteria.

Inclusion Criteria: Subjects with the American Diabetes Association ${ }^{5}(2010)$ criteria of type 2 diabetes mellitus were included in the study.

\section{Exclusion Criteria}

1. Patients with the history of alcohol intake, HBV, HCV positive patients
2. Known cases of acute and chronic liver disorder.

3. Patients with type 1 diabetes mellitus.

4. Patients with congestive cardiac failure.

5. Pregnancy.

6. Patients on drugs which can cause fatty liver. Ultrasonography was used to detect fatty liver. To make the diagnosis of NAFLD "guidelines for the assessment and management of non-alcoholic fatty liver disease in the asia pacific region" was used. Abdominal ultrasound scanning was performed using a Hitachi EZU-MT28-S1 scanner, convex EVP-C-514 probe with a 2.5-3.5 $\mathrm{MHz}$ linear transducer. The study group was divided into two groups: Type 2 diabetes mellitus patients with NAFLD and Type 2 diabetes mellitus patients without NAFLD. On selection, proper history and clinical examination were done regarding demographics, cardiovascular, hepatic and renal disease, drug use, smoking and alcohol consumption. Presence of coronary artery disease was defined by history of CAD medications, ECG changes, echocardiographic findings. Qualitative data expressed as number and percentage or proportion and analysed using chi-square test. Quantitative data will be expressed as mean \pm S.D and analysed using unpaired t-test and statistical significance will be set at $\mathrm{P}<0.05$. All statistical analysis was done using Epi info version 7.2.1.0.

\section{Observations and Results}

The randomization was adequate and there were no significant confounding factors. Out of 340 subjects of type 2 diabetes mellitus, 170 subjects $(50 \%)$ had fatty liver as detected ultrasonographically and 170 subjects (50\%) without fatty liver served as a control. The baseline physical characteristics and investigations were compared in these two groups using statistical tests of significance.

In present study among coronary risk factors hypertension was present in $19.4 \% \quad(n=33)$ in NAFLD group and $29.4 \%(n=50)$ in Non NAFLD group. Subjects with Body mass index of more than $23 \mathrm{~kg} / \mathrm{m}^{2}$ were $75.3 \%(\mathrm{n}=128)$ in NAFLD group and $64.1 \%$ (109) in Non NAFLD group. 
Subjects with Body mass index of more than $25 \mathrm{~kg} / \mathrm{m} 2$ were $41.8 \%(\mathrm{n}=71)$ in NAFLD group and $28.8 \%$ (49) in Non NAFLD group. Subjects with HBA1C of more than $7 \%$ were $75.9 \%$ $(n=129)$ in NAFLD group and $64.7 \%(n=110)$ in Non NAFLD group. Subjects with total cholesterol more than $200 \mathrm{mg} / \mathrm{dl}$ among diabetic patient with and without NAFLD were $43(25.3 \%)$ and $26(15.3 \%)$ respectively. Subjects with LDL more than $130 \mathrm{mg} / \mathrm{dl}$ among diabetic patient with and without NAFLD were 46(27.1\%) and $27(15.9 \%)$ respectively. Subjects with HDL less than $40 \mathrm{mg} / \mathrm{dl}$ among diabetic patient with and without NAFLD were 69(40.6\%) and 42(24.7\%) respectively. Subjects with triglyceride (TG) more than $150 \mathrm{mg} / \mathrm{dl}$ among diabetic patient with and without NAFLD were 63(37.1\%) and 26(15.3\%) respectively.

Table 1: Risk factors of Coronary artery disease among study groups

\begin{tabular}{|c|c|c|c|c|c|}
\hline \multirow{2}{*}{ Risk factors of CAD } & \multicolumn{2}{|c|}{ NAFLD $(\mathrm{N}=170)$} & \multicolumn{2}{|c|}{ Non NAFLD $(\mathbf{N}=170)$} & \multirow{2}{*}{ P value } \\
\hline & $\mathrm{N}$ & $\%$ & $\mathrm{~N}$ & $\%$ & \\
\hline Hypertension & 33 & 19.4 & 50 & 29.4 & 0.043 \\
\hline BMI > $23 \mathrm{~kg} / \mathrm{m}^{2}$ & 128 & 75.3 & 109 & 64.1 & 0.034 \\
\hline BMI $>25 \mathrm{~kg} / \mathrm{m}^{2}$ & 71 & 41.8 & 49 & 28.8 & 0.017 \\
\hline HbA1C>7 & 129 & 75.9 & 110 & 64.7 & 0.033 \\
\hline TC > 200mg/dl & 43 & 25.3 & 26 & 15.3 & 0.031 \\
\hline LDL >130mg/dl & 46 & 27.1 & 27 & 15.9 & 0.017 \\
\hline HDL <40mg/dl & 69 & 40.6 & 42 & 24.7 & 0.003 \\
\hline TG >150mg/dl & 63 & 37.1 & 26 & 15.3 & $<0.001$ \\
\hline
\end{tabular}

Figure 1

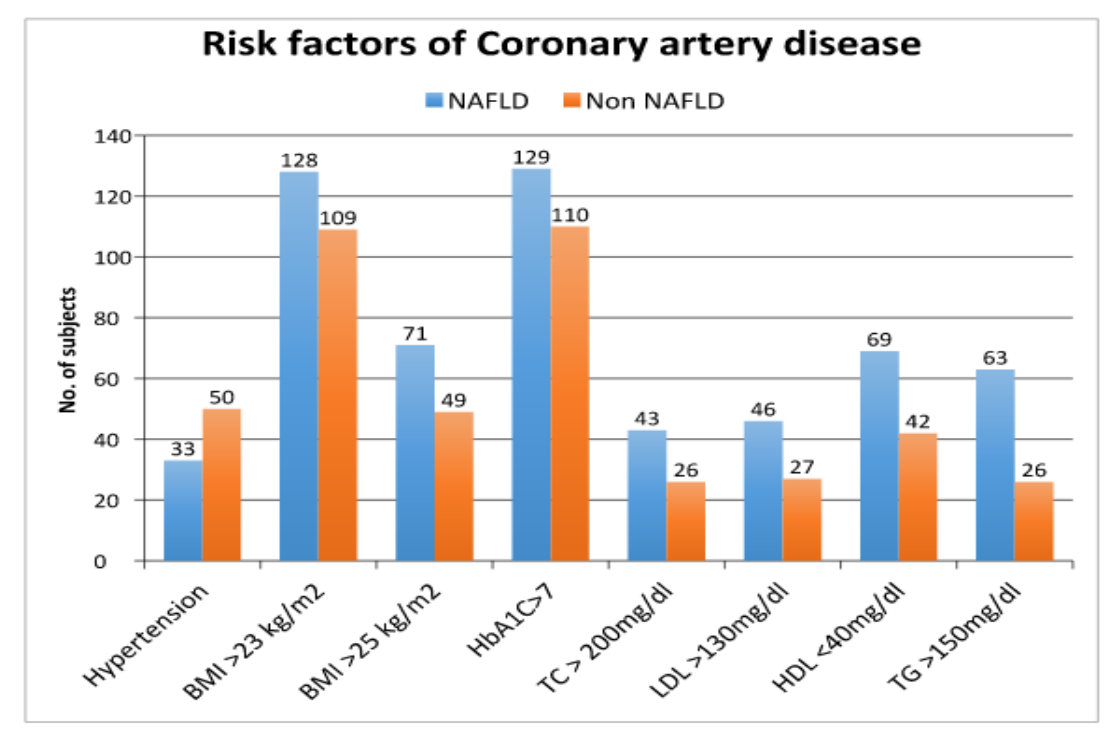

The below table showing that in NAFLD group, $39 \%$ (23 out of 59) of grade $1,70.8 \%$ (46 out of 65 ) of grade 2 and $71.7 \%$ (33 out of 46 ) of grade 3 NAFLD were having CAD. These observations

Table 2: Coronary artery disease in NAFLD diabetic patients

\begin{tabular}{|c|c|c|c|c|c|c|}
\hline \multirow{2}{*}{ NAFLD } & \multicolumn{2}{|c|}{ CAD present } & \multicolumn{2}{|c|}{ CAD Absent } & \multicolumn{2}{|c|}{ Total } \\
\hline & $\mathrm{N}$ & $\%$ & $\mathrm{~N}$ & $\%$ & $\mathrm{~N}$ & $\%$ \\
\hline Grade I & 23 & 39 & 36 & 61 & 59 & 100 \\
\hline Grade II & 46 & 70.8 & 19 & 29.2 & 65 & 100 \\
\hline Grade III & 33 & 71.7 & 13 & 28.3 & 46 & 100 \\
\hline Total & 102 & 60 & 68 & 40 & 170 & 100 \\
\hline
\end{tabular}


Figure 2

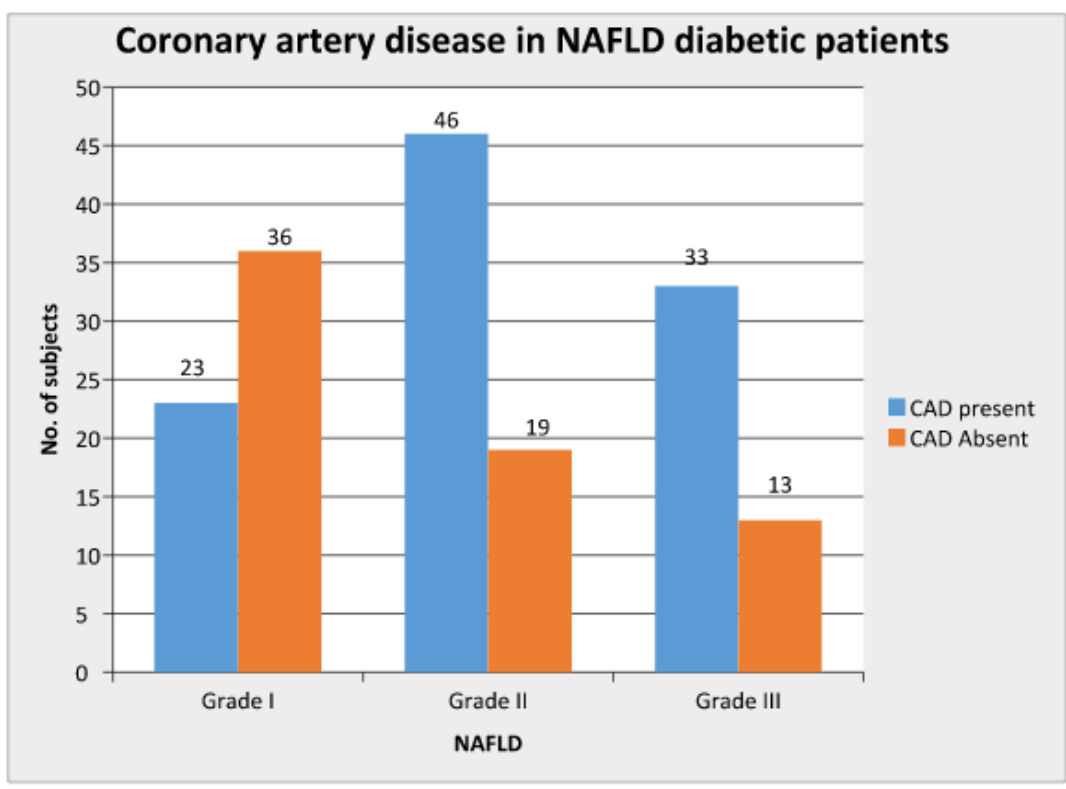

\section{Discussion}

In the present study a high prevalence of coronary artery disease risk factors was observed in type 2 diabetic subjects having NAFLD as compared patients without NAFLD and it has a strong association with severity of NAFLD similar results were reported in previous studies.

Patients were evaluated for presence of coronary risk factors like hypertension obesity, glycaemic status and dyslipidaemias among both the groups. Among coronary risk factors subjects with Body mass index of more than $23 \mathrm{~kg} / \mathrm{m} 2$ were $75.3 \%$ $(n=128)$ in NAFLD group and $64.1 \%$ (109) in Non NAFLD group ( $\mathrm{p}=0.034$ ), subjects with Body mass index of more than $25 \mathrm{~kg} / \mathrm{m} 2$ were $41.8 \%$ $(\mathrm{n}=71)$ in NAFLD group and 28.8(49) in Non NAFLD group $(\mathrm{p}=0.017)$, subjects with HBA1C of more than $7 \%$ were $75.9 \%(n=129)$ in NAFLD group and $64.7 \%(\mathrm{n}=110)$ in Non NAFLD group $(\mathrm{P}=0.033)$, subjects with total cholesterol more than 200mg/dl among diabetic patient with and without NAFLD were $43(25.3 \%)$ and 26 (15.3\%) respectively $(\mathrm{p}=0.031)$, subjects with LDL more than $130 \mathrm{mg} / \mathrm{dl}$ among diabetic patient with and without NAFLD were 46 (27.1\%) and 27 (15.9\%) respectively $(\mathrm{p}=0.017)$, subjects with HDL less than $40 \mathrm{mg} / \mathrm{dl}$ among diabetic patient with and without NAFLD were 69 (40.6\%) and $42(24.7 \%)$ respectively $(\mathrm{P}=0.003)$, subjects with triglyceride (TG) more than $150 \mathrm{mg} / \mathrm{dl}$ among diabetic patient with and without NAFLD were $63(37.1 \%)$ and 26 $(15.3 \%)$ respectively $(\mathrm{p}<0.001)$, hypertension was present in $19.4 \%(\mathrm{n}=33)$ in NAFLD group and $29.4 \%(n=50)$ in Non NAFLD group $(\mathrm{p}=0.043)$.In our study coronary risk factors were more prevalent in NAFLD group than Non NAFLD group specially for dyslipidaemia, BMI, glycaemic index but in our study hypertension and low HDL was more in Non NAFLD group.

Dyslipidaemias was associated with NAFLD. In present study higher values of total cholesterol, LDL and TG were associated with NAFLD ( $\mathrm{p}=0.031, \mathrm{p}=0.017, \mathrm{p}<0.001$ respectively). In study done by Hamaguchi $\mathrm{M}$ et $\mathrm{al}^{7}$ similar results were found and as described in earlier studies. Raminderpal Singh SibiaI et al. ${ }^{8}$ studied the prevalence of NAFLD among type 2 diabetics patients and assessed the correlation between risk factors of CAD and NAFLD in these patients. He found Hypertension $(60.7 \%$ vs $41 \%)$, microalbuminuria (36.1\% Vs $17.9 \%$ ), Metabolic syndrome $(45.9 \%$ vs $17.9 \%)$ were also more prevalent in NAFLD group than non NAFLD group. Patients in NAFLD group were also having higher systolic blood pressure at presentation, waist circumference, body mass index and 
dyslipidemia than non NAFLD group. They concluded NAFLD as a disease is fairly prevalent among type-2 diabetics and both these diseases occur simultaneously in patients with other known coronary risk factors. Hence, the presence of NAFLD can be considered as an important marker of coronary artery disease amongst type 2 diabetics. It is non-invasive, easily assessable and reproducible parameter for coronary risk stratification in type 2 diabetics. In a study Alper et al (2008) ${ }^{9}$ studied the relationship between NAFLD and angiographical severity of coronary artery disease (CAD) in patients with Metabolic Syndrome (MS). NAFLD subjects had significantly higher serum triglyceride level, waist circumference and body mass index and significantly lower HDL-cholesterol level $(\mathrm{p}<0.001)$. Coronary angiography showed significantly higher vessel $(2.5+/-0.9$ vs $1.0+/-1.0)$ and CAD severity scores $(90.2+/-40.0$ vs $36.4+/-$ $28.9)$ in patients with NAFLD $(\mathrm{p}<0.001)$. Univariate analysis revealed the presence of NAFLD $(r=0.61, p<0.001)$, grade of NAFLD $(\mathrm{r}=0.42, \mathrm{p}<0.001)$, and patient age $(\mathrm{r}=0.36$, $\mathrm{p}=0.002$ ) were significantly correlated with the CAD severity score. These results showed that presence of NAFLD is also associated with coronary risk factors.

Among T2DM patients with NAFLD, 39\% of NAFLD Grade $1,70.8 \%$ of NAFLD grade 2 and $71.7 \%$ of NAFLD grade 3 were having CAD. These observations suggest that occurrence of CAD in NAFLD patients is grade dependent as the severity of NAFLD increases the chances of occurrence of CAD also increases $(p<0.001)$.In a Korean study by DH Choi et $\mathrm{al}^{10}, 134$ Patients who underwent coronary angiography were classified into four groups by coronary artery stenosis: A, insignificant; B, one-vessel disease; $\mathrm{C}$, two-vessel disease; and $\mathrm{D}$, three-vessel disease. Ultrasonography was done to find the presence of a fatty liver and categorize fatty liver by grade: 0 , no evidence; 1 , mild; 2 , moderate; and 3 , severe. Among the 134 patients with the inclusion criteria, $82(61.2 \%)$ had ultrasonographically diagnosed NAFLD. Of the 46 patients with CAD, $37(80.4 \%)$ had evidence of a fatty liver. Groups (A vs B-D) were significantly different in terms of age, total cholesterol, triglycerides, low-density lipoprotein levels and fatty liver. Most of the fatty livers were grade 1 in group A. In the higher grade CAD group, the proportion of patients with more severe fatty livers was increased. None in group D (three-vessel disease) had a normal liver. CAD was associated with fatty liver in a gradedependent manner $(\mathrm{P}=0.025)$ as in our study.

Our study showed that there is significant relationship between presence of NAFLD and coronary artery disease and coronary risk factors in type 2 diabetes patients.

\section{Conclusions}

The findings of this study proves the association of Coronary artery disease risk factors with NAFLD and with NAFLD grading among subjects with type 2 DM. CAD factors were more associated with NAFLD group than in Non NAFLD group. Occurrence of CAD in NAFLD patients is grade dependent i.e. as the severity of NAFLD increases the chances of occurrence of CAD also increases $(\mathrm{p}<0.001)$.

The results of this study provides a prevalence pattern for Coronary artery disease risk factors in Indian Type 2 diabetes mellitus with NAFLD. There is a strong need for frequent evaluation NAFLD in type $2 \mathrm{DM}$ patients, as presence of NAFLD may be a strong predictor of future occurrence of coronary artery disease in these patients.

\section{References}

1. Afkhami Ardakani M, Rashidi M. Risk factors of type 2 diabetes. Journal of Rafsanjan University of Medical Sciences 2005;4(4):348-65 [In Persian].

2. Farrell GC, Larter CZ. Nonalcoholic Fatty Liver Disease: from Steatosis to cirrhosis Hepatology 2006; 43:S00-S112.

3. Garcia-Monzon C., Martin-Perez E., Iacono O.L., Fernandez-Bermejo M., 
Majano P.L., Apolinario A., Larranaga E., Moreno-Otero R., Characterization of pathogenic and prognostic factors of nonalcoholic steato-hepatitis associated with obesity, J Hepatol,2000,33(5):716-724.

4. Hamaguchi $\mathrm{M}$, Kojima $\mathrm{T}$, Takeda $\mathrm{N}$ etal.The metabolic syndrome as a predictor of Non-alcoholic fatty liver disease. Ann. Intern. Med. 2005 ; 143:722-816.

5. "Diagnosis and classification of diabetes mellitus". Diabetes Care (American Diabetes Association). 33 Suppl 1 (Supplement_1): S62-9. January 2010. doi:10.2337/dc10-S062.PMC 2797383. PMID 20042775.

6. Wolfs MGM, Gruben N, Rensen SS, Verdam FJ, Greve JW, Driessen A, et al.Determining the association between adipokine expression in multiple tissues and phenotypic features of non-alcoholic fatty liver disease in obesity. Nutr Diabetes 2015;5.

7. Hamaguchi M, Kojima T, Takeda N, Nagata C, Takeda J, [12]Sarui H, et al. Nonalcoholic fatty liver disease is a novel predictor of cardiovascular disease. World J Gastroenterol. 2007;13(10):1579-84.

8. Raminderpal Singh Sibia, Sandeep Dhoot, et al. Prevalence of Non-Alcoholic Fatty Liver Disease in Patients with Type 2 Diabetes and its Correlation with Coronary Risk Factors DOI: 10.7860/ NJLM/2016/ 20612:2168.

9. Alper AT, Hasdemir H, Sahin S, et al. The relationship between nonalcoholic fatty liver disease and the severity of coronary artery disease : Coronary Artery Disease 2007; 8: 433-6.

10. Dae Hee Choi et al. Nonalcoholic fatty liver disease is associated with coronary artery disease in Koreans World $\mathrm{J}$ Gastroenterol. 2013 Oct 14; 19(38): 64536457. 